\title{
WRONSKIAN REPRESENTATIONS OF HYPERGEOMETRIC INTEGRALS
}

\author{
ANTHONY ZUCCOLO ${ }^{\dagger}$ \\ INDIANA UNIVERSITY NORTHWEST, GARY \\ ADVISOR: AXEL SCHULZE-HALBERG ${ }^{\ddagger}$ \\ INDIANA UNIVERSITY NORTHWEST, GARY
}

\begin{abstract}
We construct new representations of hypergeometric integrals in terms of Kampé de Fériet functions. Our method is based on recently developed integral formulas that allow to express certain integrals through Wronskians.
\end{abstract}

MSC Primary 33C60; Secondary 33C15;

Key words: integral formula, hypergeometric function, Wronskian

\section{Introduction}

One of the standing issues in calculus and its applications is the closed-form resolution of integrals. While numerical methods allow to determine any definite integral to arbitrary precision, these methods are essentially restricted to integrals that do not involve unknown parameters. Furthermore, in many applications it is useful to have a closed-form expression of the integral that can be processed further before evaluating at particular numerical limits. Since only few integrals admit their resolution in closed-form, it is desirable to have resources at hand for finding them. Known integrals are tabulated in books, such as [1] [9] [15], and they can also be found using online databases [16]. A particularly important integrals class are integrals of special functions [5] [11]. Such functions arise as solutions of linear differential equations that govern systems in applied mathematics, physics, economics, engineering, and many other fields. Special functions cannot be represented through a composition of elmentary functions (such as exponentials and powers), but are typically defined by means of integrals or power series. Due to this property it is very difficult to resolve integrals of special functions in closed form, such that very few of them are known and tabulated in the aforementioned resources. It is therefore desirable to develop methods that can resolve integrals of special functions. In this work we will present such a method, together with applications that focus on a particular kind of special functions, called hypergeometric functions [1]. These functions are especially important in quantum physics, where they describe the behaviour of many fundamental systems, see for example [13] and references therein. Hypergeometric functions contain many special and elementary functions as particular cases [16], such as elliptic integrals and several types of orthogonal

Date: xyz, 2018.

${ }^{\dagger}$ Electronic Address: amz18@sbcglobal.net.

${ }^{\ddagger}$ Electronic Address: axgeschu@iun.edu. 
polynomials. In order to construct closed-form integrals of hypergeometric functions, we use a recently developed method [6] [12] that arises from the supersymmetry formalism in quantum mechanics. This method allows to express certain single and multiple integrals of special functions in terms of their Wronskians. In the single-variable case the domain of integration is arbitrary, such that we essentially obtain a closed-form representation of the indefinite integral. While multiple integration is limited to triangular domains, two of the vertices can be chosen arbitrarily. The remainder of this work is organized as follows. In section 2 we introduce Kampé de Fériet functions that we will use in subsequent sections. Section 3 summarizes basic facts about two formulas for the resolution of single and double integrals that were obtained in [6] [12]. In the following sections 3 and 4 we apply our formulas to integrals of confluent hypergeometric and hypergeomtric type, respectively.

\section{Kampé de Fériet functions}

One of the key concepts that we use throughout this work is the Kampé de Fériet (KdF) function that generalizes hypergeometric functions in two variables. While a detailed discussion of these functions and their properties can be found in [8] [14] [7], the purpose of this section is to state their definition and present relationships with partial derivatives of certain hypergeometric functions. In the following we introduce several sets of parameters, using the notation $\left(\alpha_{p}\right)$ for the ordered list $\left(\alpha_{1}, \ldots, \alpha_{p}\right)$ of parameters and $\left(\left(\alpha_{p}\right)\right)_{n}$ for the product of Pochhammer symbols $\left(\alpha_{1}\right)_{n} \ldots\left(\alpha_{p}\right)_{n}$, where $\left(\left(\alpha_{0}\right)\right)_{n}=1$. A KdF function is formally defined as

$$
F_{q: s ; v}^{p: r ; u}\left[\begin{array}{c}
\left(a_{p}\right):\left(c_{r}\right) ;\left(f_{u}\right) \\
\left(b_{q}\right):\left(d_{s}\right) ;\left(g_{v}\right)
\end{array} \mid x, y\right]=\sum_{m=0}^{\infty} \sum_{n=0}^{\infty} \frac{\left(\left(a_{p}\right)\right)_{m+n}\left(\left(c_{r}\right)\right)_{m}\left(\left(f_{u}\right)\right)_{n}}{\left(\left(b_{q}\right)\right)_{m+n}\left(\left(d_{s}\right)\right)_{m}\left(\left(g_{v}\right)\right)_{n}} \frac{x^{m} y^{n}}{m ! n !},
$$

where $x$ and $y$ are complex numbers. This double series can be interpreted as a generalization of the two-variable hypergeometric function. Convergence criteria for the series are established in [8] [14]. In this note we will use $\mathrm{KdF}$ functions to represent partial derivatives of hypergeometric functions that are taken with respect to their parameter arguments. Since covering arbitraryorder derivatives for general hypergeometric functions is beyond the scope of this work, we restrict ourselves to stating the first-order partial derivatives of the confluent hypergeometric function ${ }_{1} F_{1}$ and the hypergeometric function ${ }_{2} F_{1}$ in terms of KdF functions. For details on the construction of the following identities, the reader may refer to [2]-[4].

$$
\begin{aligned}
& \frac{\partial}{\partial a}{ }_{1} F_{1}(a, b, z)=\frac{z}{b} F_{2: 1 ; 1}^{1: 2 ; 1}\left[\begin{array}{c}
a+1: a, 1 ; 1 \\
b+1,2: a+1 ; 1
\end{array} \mid z, z\right] \\
& \frac{\partial}{\partial b}{ }_{1} F_{1}(a, b, z)=-\frac{a z}{b^{2}} F_{2: 1 ; 1}^{1: 2 ; 1}\left[\begin{array}{c}
a+1: b, 1 ; 1 \\
b+1,2: b+1 ; 1
\end{array} \mid z, z\right] \\
& \frac{\partial}{\partial a}{ }_{2} F_{1}(a, b, c, z)=\frac{b z}{c} F_{2: 1 ; 1}^{2: 1 ; 2}\left[\begin{array}{ccc|c}
a+1, b+1: & 1 ; a, 1 \\
c+1,2 & : a+1 ; & 1
\end{array} \mid z, z\right] \\
& \frac{\partial}{\partial b}{ }_{2} F_{1}(a, b, c, z)=-\frac{a b z}{c^{2}} F_{2: 1 ; 1}^{2: 1 ; 2}\left[\begin{array}{ccc}
a+1, b+1: & 1 & ; b, 1 \\
c+1,2 & : b+1 ; 1
\end{array} \mid z, z\right]
\end{aligned}
$$

These identities can also be used to represent derivatives of other hypergeometric functions, such as the confluent hypergeometric function of the second kind or Tricomi function. We will comment on this aspect in more detail below. 


\section{Wronskian integral formulas}

We will now introduce the main tool that is used throughout this work, a particular kind of integral formula that resolves certain integrals in terms of Wronskians. For real numbers $a, b, \alpha, \beta$ that satisfy the constraints $a<b$ and $\alpha<\beta$, we define the set $D \subset \mathbb{R}^{2}$ as the rectangular region $D=(a, b) \times(\alpha, \beta)$. Furthermore, we assume that the functions $u$ and $v$ are smooth, linearly independent solutions of the differential equation

$$
\frac{\partial^{2}}{\partial x^{2}} y(x, \lambda)+[\lambda-V(x)] y(x, \lambda)=0, \quad(x, \lambda) \in D,
$$

where $V$ is continuous on $(a, b)$. Under these assumptions, certain integrals of the solutions admit a representation in terms of Wronskians [6] [12]. As a consequence, this representation allows for integration through mere differentiation. Starting out with single-variable integration, for $x_{0}, x \in(a, b)$ and $(x, \lambda) \in D$ we have the identity [6]

$$
\int_{x_{0}}^{x} u(t, \lambda)^{2} d t=\left.W_{u, \frac{\partial u}{\partial \lambda}}(t, \lambda)\right|_{x} ^{x_{0}}=W_{u, \frac{\partial u}{\partial \lambda}}\left(x_{0}, \lambda\right)-W_{u, \frac{\partial u}{\partial \lambda}}(x, \lambda),
$$

provided the integral on the left side exists. Note that the symbol $W$ stands for the Wronskian of the functions in its index. Its should be clarified here that the variable of differentiation in the Wronskian is $x$. More precisely, we have

$$
W_{u, \frac{\partial u}{\partial \lambda}}(x, \lambda)=u(x, \lambda) \frac{\partial u(x, \lambda)}{\partial x \partial \lambda}-\frac{\partial u(x, \lambda)}{\partial \lambda} \frac{\partial u(x, \lambda)}{\partial x} .
$$

The counterpart of formula (7) for double integration reads

$$
\begin{aligned}
& \int_{x_{0}}^{x} \int_{x_{0}}^{x_{1}}\left[\frac{u\left(x_{2}, \lambda\right)}{u\left(x_{1}, \lambda\right)}\right]^{2} d x_{2} d x_{1}= \\
& \quad=\frac{1}{u\left(x_{0}, \lambda\right)} \frac{\partial}{\partial \lambda} u\left(x_{0}, \lambda\right)-\frac{1}{u(x, \lambda)} \frac{\partial}{\partial \lambda} u(x, \lambda)+W_{u, \frac{\partial u}{\partial \lambda}}\left(x_{0}, \lambda\right) \int_{x_{0}}^{x} \frac{1}{u(t, \lambda)^{2}} d t .
\end{aligned}
$$

We observe that the domain of integration is a right triangular region in the $x_{1}-x_{2}$-plane. The result (8) that was obtained in [12], can be slightly improved for our purposes. In particular, we want to remove the single-variable integral from the right side of (8) in order to have an integral-free representation. To this end, we recall that the solutions $u$ and $v$ of (6) are linked through the reduction-of-order formula. Taking into account our integration limits and the fact that the Wronskian of $u$ and $v$ is not necessarily equal to one, this formula can be written as

$$
\frac{1}{W_{u, v}(\lambda)}\left[\frac{v(x, \lambda)}{u(x, \lambda)}-\frac{v\left(x_{0}, \lambda\right)}{u\left(x_{0}, \lambda\right)}\right]=\int_{x_{0}}^{x} \frac{1}{u(t, \lambda)^{2}} d t .
$$

Note that the Wronskian $W_{u, v}(\lambda)$ does not depend on $x$ or $x_{0}$ [10]. Now, upon substitution of (9) into the identity (8), we obtain the final result

$$
\begin{aligned}
& \int_{x_{0}}^{x} \int_{x_{0}}^{x_{1}}\left[\frac{u\left(x_{2}, \lambda\right)}{u\left(x_{1}, \lambda\right)}\right]^{2} d x_{2} d x_{1}= \\
& =\frac{1}{u\left(x_{0}, \lambda\right)} \frac{\partial}{\partial \lambda} u\left(x_{0}, \lambda\right)-\frac{1}{u(x, \lambda)} \frac{\partial}{\partial \lambda} u(x, \lambda)+\frac{W_{u, \frac{\partial u}{\partial \lambda}}\left(x_{0}, \lambda\right)}{W_{u, v}(\lambda)}\left[\frac{v(x, \lambda)}{u(x, \lambda)}-\frac{v\left(x_{0}, \lambda\right)}{u\left(x_{0}, \lambda\right)}\right] .
\end{aligned}
$$


We observe that the right side of this relation does not contain any integral anymore, such that the double integral on its left side is expressed entirely through partial derivatives. We further remark that the identities (7) and (10) can be generalized to multiple integration of arbitrary even order [12], which is beyond the scope of this work.

\section{Confluent hypergeometric integrals}

We will now demonstrate how specific integral formulas for confluent hypergeometric functions can be constructed from (7) and (10). To this end, we need to find functions $u$ and $v$ that are of confluent hypergeometric type and satisfy a differential equation of the form (6). A straightforward approach to finding such functions is to transform the confluent hypergeometric equation into the shape (6). Depending on the choice of transformation we obtain an infinite class of functions that solve an equation of the latter type. Hence, let us start out by considering the confluent hypergeometric equation [1]

$$
z \psi^{\prime \prime}(z)+(b-z) \psi^{\prime}(z)-a \psi(z)=0,
$$

where $a, b$ are real-valued constants. The general solution of (11) can be written in the form [1]

$$
\psi(z)=c_{1}{ }_{1} F_{1}(a, b, z)+c_{2} U(a, b, z)
$$

provided $b$ is not a nonpositive integer. Here, ${ }_{1} F_{1}$ and $U$ denote confluent hypergeometric functions of the first and second kind, respectively. Furthermore, $c_{1}$ and $c_{2}$ stand for arbitrary constants. In order to apply our integral formulas (7) and (10), we must bring equation (11) to the form (6). To this end, let us introduce

$$
y(x)=\sqrt{\frac{1}{z^{\prime}(x)}} z(x)^{\frac{b}{2}} \exp \left[-\frac{z(x)}{2}\right] \psi[z(x)],
$$

where the coordinate change $z$ is a smooth function and we assume that $\psi$ solves (11). The function (13) is a solution of the equation

$$
y^{\prime \prime}(x)+W(x) y(x)=0,
$$

where $W$ is given by the expression

$$
W(x)=\frac{z^{\prime}(x)^{2}}{z(x)}\left[-a+\frac{b}{2}-\frac{b^{2}}{4 z(x)}+\frac{b}{2 z(x)}-\frac{z(x)}{4}\right]-\frac{3 z^{\prime \prime}(x)^{2}}{4 z^{\prime}(x)^{2}}+\frac{z^{\prime \prime \prime}(x)}{2 z^{\prime}(x)} .
$$

The transformation (13) renders our general solution (12) in the form

$$
y(x)=\sqrt{\frac{1}{z^{\prime}(x)}} z(x)^{\frac{b}{2}} \exp \left[-\frac{z(x)}{2}\right]\left\{c_{1}{ }_{1} F_{1}[a, b, z(x)]+c_{2} U[a, b, z(x)]\right\} .
$$

It is important to observe that (14) does not match (6) yet because the parameter $\lambda$ has not been defined. In order to do so, we must choose the coordinate change $z$ in such a way that a constant additive term is generated in (15). More precisely, inspection of (6) shows that the required form is $W=\lambda-V$ for a constant $\lambda$ and a function $V$. This form is only attained if the coordinate change $z$ is chosen suitably. Since there are infinitely many ways of obtaining this form, we restrict ourselves to some examples. 


\subsection{Single integration}

Let us first present an example for our integral formula (7) by choosing the particular coordinate change

$$
z(x)=-\frac{x^{2}}{4}
$$

Upon substitution into (15) we must obtain an expression that contains an additive constant. After performing the substitution and renaming $a=\lambda$, the function $W$ takes the form

$$
W(x)=\lambda-\frac{b}{2}-\frac{x^{2}}{16}-\frac{3}{4 x^{2}}+\frac{2 b-b^{2}}{x^{2}},
$$

As desired, the right side contains a constant term. We can therefore match the latter expression with the general form $W=\lambda-V$ from (6), if we choose

$$
V(x)=\frac{b}{2}+\frac{x^{2}}{16}+\frac{3}{4 x^{2}}-\frac{2 b-b^{2}}{x^{2}} .
$$

Consequently, our identity (7) is applicable to the present example. It remains to determine the function $u$ in the latter identity. This function is simply the solution of (6) associated with (18), that we can obtain by plugging (17) into (16). We get

$$
y(x)=\exp \left(\frac{x^{2}}{8}\right) x^{b-\frac{1}{2}}\left[c_{1}{ }_{1} F_{1}\left(\lambda, b,-\frac{1}{4} x^{2}\right)+c_{2} U\left(\lambda, b,-\frac{x^{2}}{4}\right)\right] .
$$

Before we continue, let us briefly make a statement about the monomial term on the right side of (19). Taking into account the setting (17), the latter monomial term is obtained as follows

$$
\sqrt{\frac{1}{z^{\prime}(x)}} z(x)^{\frac{b}{2}}=(-1)^{\frac{1}{2}} 4^{-\frac{b}{2}} x^{b-\frac{1}{2}}
$$

Since we are merely interested in solutions of equation (16), irrelevant constant factors can be discarded. This is why in (19) we only retain the monomial term without the constants. For the sake of simplicity let us now incorporate the additional settings $c_{1}=1, c_{2}=0$. After renaming $y(x)=u(x, \lambda)$, we obtain from (19)

$$
u(x, \lambda)=\exp \left(\frac{x^{2}}{8}\right) x^{b-\frac{1}{2}}{ }_{1} F_{1}\left(\lambda, b,-\frac{x^{2}}{4}\right) .
$$

We are now ready to apply our identity (7) to this function. In order to do so, we will evaluate the right side of the latter identity. Evaluation of the Wronskian gives the following result

$$
\begin{aligned}
W_{u, \frac{\partial u}{\partial \lambda}}(x, \lambda) & =\frac{x^{2 b}}{2 b} \exp \left(\frac{x^{2}}{4}\right)\left\{\lambda_{1} F_{1}\left(\lambda+1, b+1,-\frac{x^{2}}{4}\right) \frac{\partial}{\partial \lambda}{ }_{1} F_{1}\left(\lambda, b,-\frac{x^{2}}{4}\right)\right. \\
& \left.-\lambda_{1} F_{1}\left(\lambda, b,-\frac{x^{2}}{4}\right) \frac{\partial}{\partial \lambda}{ }_{1} F_{1}\left(\lambda+1, b+1,-\frac{x^{2}}{4}\right)\right\} .
\end{aligned}
$$

We observe that this expression contains two partial derivatives with respect to the first argument of the confluent hypergeometric function. These derivatives can now be replaced by means of 
the identity (2). We obtain the result

$$
\begin{aligned}
& W_{u, \frac{\partial u}{\partial \lambda}}(x, \lambda)=-\frac{x^{2 b}}{2 b} \exp \left(\frac{1}{4} x^{2}\right)\left\{{ }_{1} F_{1}\left(\lambda, b,-\frac{x^{2}}{4}\right){ }_{1} F_{1}\left(\lambda+1, b+1,-\frac{x^{2}}{4}\right)\right. \\
& -\lambda_{1} F_{1}\left(\lambda+1, b+1,-\frac{x^{2}}{4}\right)\left[-\frac{x^{2}}{4 b}\right] F_{2: 1 ; 1}^{1: 2 ; 1}\left[\begin{array}{c}
\lambda+1: \lambda, 1 ; 1 \\
b+1,2: \lambda+1 ; 1
\end{array} \mid-\frac{x^{2}}{4},-\frac{x^{2}}{4}\right] \\
& \left.+\lambda_{1} F_{1}\left(\lambda, b,-\frac{x^{2}}{4}\right)\left[-\frac{x^{2}}{4 b}\right] F_{2: 1 ; 1}^{1: 2 ; 1}\left[\begin{array}{c}
\lambda+2: \lambda+1,1 ; 1 \\
b+2,2: \lambda+2 ; 1
\end{array} \mid-\frac{x^{2}}{4},-\frac{x^{2}}{4}\right]\right\} .
\end{aligned}
$$

Upon plugging the function (20) and the Wronskian (21) into the integral formula (7), we obtain the overall result

$$
\begin{gathered}
\int_{x_{0}}^{x} \exp \left(\frac{t^{2}}{4}\right) t^{2 b-1}{ }_{1} F_{1}\left(\lambda, b,-\frac{t^{2}}{4}\right)^{2} d t= \\
=\frac{t^{2 b}}{2 b} \exp \left(\frac{t^{2}}{4}\right)\left\{{ }_{1} F_{1}\left(\lambda, b,-\frac{t^{2}}{4}\right){ }_{1} F_{1}\left(\lambda+1, b+1,-\frac{t^{2}}{4}\right)\right. \\
-\lambda_{1} F_{1}\left(\lambda+1, b+1,-\frac{t^{2}}{4}\right)\left[-\frac{t^{2}}{4 b}\right] F_{2: 1 ; 1}^{1: 2 ; 1}\left[\begin{array}{c}
\lambda+1: \lambda, 1 ; 1 \\
b+1: \lambda+1 ; 1
\end{array} \mid-\frac{t^{2}}{4},-\frac{t^{2}}{4}\right] \\
\left.+\lambda_{1} F_{1}\left(\lambda, b,-\frac{t^{2}}{4}\right)\left[-\frac{t^{2}}{4 b}\right] F_{2: 1 ; 1}^{1: 2 ; 1}\left[\begin{array}{c}
\lambda+2: \lambda+1,1 ; 1 \\
b+2,2: \lambda+2 ; 1
\end{array} \mid-\frac{t^{2}}{4},-\frac{t^{2}}{4}\right]\right\}\left.\right|_{x_{0}} ^{x},
\end{gathered}
$$

This identity provides a closed-form evaluation of the confluent hypergemetric integral on its left side in terms of Kampé de Fériet functions. To the best of our knowledge, this result has not been stated before in the literature. Now, observe that we picked a particularly simple $u$ from (19) by setting $c_{2}=0$, which removes the confluent hypergeometric function of the second kind. This is no restriction to our formula (7), as we will see now. Suppose that in (19) we employ the opposite setting $c_{1}=0$, replacing ${ }_{1} F_{1}$ by $U$ on the left side of $(23)$. Using the known link between the confluent hypergeometric functions [1], we get

$$
\begin{aligned}
& \int_{x_{0}}^{x} \exp \left(\frac{t^{2}}{4}\right) t^{2 b-1} U\left(\lambda, b,-\frac{t^{2}}{4}\right)^{2} d t= \\
& \quad=\int_{x_{0}}^{x} \exp \left(\frac{t^{2}}{4}\right) t^{2 b-1}\left[\frac{\Gamma(b-1)}{\Gamma(\lambda)}\left(-\frac{t^{2}}{4}\right)^{1-b}{ }_{1} F_{1}\left(\lambda-b+1,2-b,-\frac{t^{2}}{4}\right)\right. \\
& \left.+\frac{\Gamma(1-b)}{\Gamma(\lambda-b+1)}{ }_{1} F_{1}\left(\lambda, b,-\frac{t^{2}}{4}\right)\right]^{2} d t
\end{aligned}
$$

After multiplying out the brackets we can split the integral into three parts, each of which allows for the application of (7) and subsequent replacement of the derivative through KdF functions. Since the calculation and the resulting expressions will be very long, we omit to show them here. 
Example: elementary case. Although the purpose of (23) is the construction of integrals that contain confluent hypergeometric functions, there are many special cases of parameters settings that render the integral in (23) elementary. Let us employ the choice $\lambda=-1, b=$ $1 / 4, x_{0}=0$ and $x=1$. We get

$$
\begin{aligned}
& \int_{0}^{1} \frac{\left(t^{2}+1\right)^{2}}{\sqrt{t}} \exp \left(\frac{t^{2}}{4}\right) d t=4 \exp \left(\frac{1}{4}\right)-2 \exp \left(\frac{1}{4}\right) F_{2: 1 ; 1}^{1: 2 ; 1}\left[\begin{array}{rrr}
0 & :-1,1 ; 1 \\
\frac{5}{4}, 2: & 0 & ; 1
\end{array} \mid-\frac{1}{4},-\frac{1}{4}\right]
\end{aligned}
$$

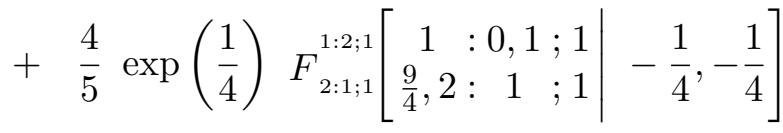

$$
\begin{aligned}
& =-4 \exp \left(-\frac{1}{4}\right)+(8-8 i) \Gamma\left(\frac{5}{4}\right)-2 \mathrm{E}_{\frac{3}{4}}\left(-\frac{1}{4}\right) \text {, }
\end{aligned}
$$

where E stands for the exponential integral. This result can be verified by direct evaluation of integral in (23).

Example. In this example we present another particular case of the integral on the left side of (23). Our goal is to derive an alternative representation of the following identity that can be found in [9]. For any real $\lambda$ we have

$$
\begin{aligned}
& \int_{0}^{\infty} \exp (-s u) u^{b-1}{ }_{1} F_{1}(\lambda, b, u)^{2} d u=\frac{\Gamma(b) s^{2 \lambda-b}}{(s-1)^{2 \lambda}}{ }_{2} F_{1}\left(\lambda, \lambda, b, \frac{1}{(s-1)^{2}}\right), \\
& \text { provided } \operatorname{Re}(b)>0 \text { and } \operatorname{Re}(s)>2 \text {. }
\end{aligned}
$$

Before we continue, let us focus on the particular case of this identity that arises if $\lambda$ is a negative integer or zero. In this situation (24) holds for all values of $s$ that satisfy $\operatorname{Re}(s)>0$. This is so because the confluent hypergeometric function in the integral of (24) degenerates to a Laguerre polynomial of degree $\lambda$. We observe that in the special case $s=1$ the right side of (24) becomes undefined. While it is possible to retain its form by interpreting it in the sense of a limit $s \rightarrow 1$, we will now use our previous result (23) to find an alternative form of (24) for the case $s=1$. Recall that during the subsequent calculations in this paragraph we need to make the standing assumption that $\lambda$ is a nonpositive integer. We start out by substituting $u=-t^{2} / 4$ in the latter integral, which gives

$$
\int_{x_{0}}^{x} \exp (-u) u^{b-1}{ }_{1} F_{1}(\lambda, b, u)^{2} d u=-\frac{1}{2}\left(-\frac{1}{4}\right)^{b-1} \int_{2 i \sqrt{x_{0}}}^{2 i \sqrt{x}} \exp \left(\frac{t^{2}}{4}\right) t^{2 b-2}{ }_{1} F_{1}\left(\lambda, b,-\frac{t^{2}}{4}\right)^{2} d t
$$

We note that the integral on the left side is real-valued. Thus, the same must be true for the integral on the right side of (25), despite its integration interval being on the imaginary axis. While the exponential and the hypergeometric function have real-valued arguments, we must impose the following convention on the monomial

$$
t^{2 b-2}=\left(t^{2}\right)^{b-1} .
$$

Thus, an imaginary value of $t$ will result in a real-value of the monomial. Now, we observe that up to constant factors and integration limits, the new integral (25) coincides with its counterpart 
on the left side of our formula (23). Combination of this formula and (25) gives the result

$$
\begin{aligned}
\int_{x_{0}}^{x} \exp (-u) u^{b-1}{ }_{1} F_{1}(\lambda, b, u)^{2} d u & \\
& =\frac{u^{b}}{b} \exp (-u)\left\{{ }_{1} F_{1}(\lambda, b, u)_{1} F_{1}(\lambda+1, b+1, u)\right. \\
& -\frac{\lambda u}{b}{ }_{1} F_{1}(\lambda+1, b+1, u) F_{2: 1 ; 1}^{1: 2 ; 1}\left[\begin{array}{c}
\lambda+1: \lambda+1,2: \lambda+1 ; 1 \\
b+1
\end{array} \mid u, u\right] \\
& \left.+\frac{\lambda u}{b}{ }_{1} F_{1}(\lambda, b, u) F_{2: 1 ; 1}\left[\begin{array}{c}
\lambda+2: \lambda+1,1 ; 1 \\
b+2,2: \lambda+2 ; 1
\end{array} \mid u, u\right]\right\}\left.\right|_{2 i \sqrt{x_{0}}} ^{2 i \sqrt{x}}
\end{aligned}
$$

This is a closed-form representation of the integral in (24) for $s=1$ and arbitrary integration limits. Before we state our result, let us note that the right side of (26) vanishes for $u=0$, while its first term becomes zero as $u$ goes to infinity. Taking into account these simplifications, we obtain the limit representation

$$
\begin{aligned}
& \int_{0}^{\infty} \exp (-u) u^{b-1}{ }_{1} F_{1}(\lambda, b, u)^{2} d u= \\
& \quad=\lim _{x \rightarrow \infty} \frac{\lambda(-4 x)^{\frac{b}{2}+1}}{b^{2}} \exp (-2 i \sqrt{x}) \\
& \quad \times\left\{{ }_{1} F_{1}(\lambda+1, b+1,2 i \sqrt{x}) F_{2: 1 ; 1}^{1: 2 ; 1}\left[\begin{array}{c}
\lambda+1: \lambda, 1 ; 1 \\
b+1,2: \lambda+1 ; 1
\end{array} \mid 2 i \sqrt{x}, 2 i \sqrt{x}\right]\right. \\
& \left.\quad+{ }_{1} F_{1}(\lambda, b, 2 i \sqrt{x}) F_{2: 1 ; 1}^{1: 2 ; 1}\left[\begin{array}{c}
\lambda+2: \lambda+1,1 ; 1 \\
b+2,2: \lambda+2 ; 1
\end{array} \mid 2 i \sqrt{x}, 2 i \sqrt{x}\right]\right\} .
\end{aligned}
$$

This representation complements identity (24) for $s=1$, assuming that $\lambda$ is a nonpositive integer. As a final remark let us mention that in the latter case we can rewrite identity (24) by expressing the confluent hypergeometric and the hypergeometric functions on the left and the right side by Laguerre and Jacobi polynomials, respectively.

\subsection{Double integration}

Let us now construct a formula involving double integration of confluent hypergeometric functions by means of our identity (10). For the sake of simplicity, we will again make use of the function (20). We will obtain a closed-form expression for the double integral in (10) by evaluating its right side. To this end, we need to provide a function $v$ that solves equation (14), such that (20) and $v$ are linearly independent. We can obtain such a function from (16) by setting $c_{1}=0$ and $c_{2}=1$. This gives

$$
v(x, \lambda)=\exp \left(\frac{x^{2}}{8}\right) x^{b-\frac{1}{2}} U\left(\lambda, b,-\frac{x^{2}}{4}\right) .
$$


Upon substitution of (20) and (27) into the right side of (10), we get the following result

$$
\begin{aligned}
\frac{1}{u\left(x_{0}, \lambda\right)} & \frac{\partial}{\partial \lambda} u\left(x_{0}, \lambda\right)-\frac{1}{u(x, \lambda)} \frac{\partial}{\partial \lambda} u(x, \lambda)+\frac{W_{u, \frac{\partial u}{\partial \lambda}}\left(x_{0}, \lambda\right)}{W_{u, v}(\lambda)}\left[\frac{v(x, \lambda)}{u(x, \lambda)}-\frac{v\left(x_{0}, \lambda\right)}{u\left(x_{0}, \lambda\right)}\right] \\
= & \frac{\frac{\partial}{\partial \lambda}{ }_{1} F_{1}\left(\lambda, b,-\frac{x_{0}^{2}}{4}\right)}{{ }_{1} F_{1}\left(\lambda, b,-\frac{x_{0}^{2}}{4}\right)}-\frac{\frac{\partial}{\partial \lambda}{ }_{1} F_{1}\left(\lambda, b,-\frac{x^{2}}{4}\right)}{{ }_{1} F_{1}\left(\lambda, b,-\frac{x^{2}}{4}\right)} \\
+ & \frac{x_{0}^{2 b} \Gamma(\lambda+1) \exp \left(\frac{x_{0}^{2}}{4}\right)}{2 b(-1)^{b} 2^{2 b-1} \lambda \Gamma(b){ }_{1} F_{1}\left(\lambda, b,-\frac{x^{2}}{4}\right){ }_{1} F_{1}\left(\lambda, b,-\frac{x_{0}^{2}}{4}\right)} \\
\times & {\left[{ }_{1} F_{1}\left(\lambda, b,-\frac{x^{2}}{4}\right) U\left(\lambda, b,-\frac{x_{0}^{2}}{4}\right)-{ }_{1} F_{1}\left(\lambda, b,-\frac{x_{0}^{2}}{4}\right) U\left(\lambda, b,-\frac{x^{2}}{4}\right)\right] } \\
\times & {\left[{ }_{1} F_{1}\left(\lambda, b,-\frac{x_{0}^{2}}{4}\right){ }_{1} F_{1}\left(\lambda+1, b+1,-\frac{x_{0}^{2}}{4}\right)-\lambda{ }_{1} F_{1}\left(\lambda+1, b+1,-\frac{x_{0}^{2}}{4}\right)\right.} \\
\times & \left.\frac{\partial}{\partial \lambda}{ }_{1} F_{1}\left(\lambda, b,-\frac{x_{0}^{2}}{4}\right)+\lambda{ }_{1} F_{1}\left(\lambda, b,-\frac{x_{0}^{2}}{4}\right) \frac{\partial}{\partial \lambda}{ }_{1} F_{1}\left(\lambda+1, b+1,-\frac{x_{0}^{2}}{4}\right)\right] .
\end{aligned}
$$

This expression contains derivatives of the function ${ }_{1} F_{1}$ that we can replace using the rules (2) and (3). After making the replacements and plugging (20) into the left side of (10), the latter identity takes its final form

$$
\begin{aligned}
& \int_{x_{0}}^{x} \int_{x_{0}}^{x_{1}} \exp \left(\frac{x_{2}^{2}}{4}-\frac{x_{1}^{2}}{4}\right) x_{1}^{1-2 b} x_{2}^{2 b-1}\left[\frac{{ }_{1} F_{1}\left(\lambda, b,-\frac{x_{2}^{2}}{4}\right)}{{ }_{1} F_{1}\left(\lambda, b,-\frac{x_{1}^{1}}{4}\right)}\right]^{2} d x_{2} d x_{1} \\
& =\frac{1}{{ }_{1} F_{1}\left(\lambda, b,-\frac{x_{0}^{2}}{4}\right)}\left[-\frac{x_{0}^{2}}{4 b}\right] F_{2: 1 ; 1}^{1: 2 ; 1}\left[\begin{array}{c}
\lambda+1: \lambda, 1 ; 1 \\
b+1,2: \lambda+1 ; 1
\end{array} \mid-\frac{x_{0}^{2}}{4},-\frac{x_{0}^{2}}{4}\right] \\
& -\frac{1}{{ }_{1} F_{1}\left(\lambda, b,-\frac{x^{2}}{4}\right)}\left[-\frac{x_{0}^{2}}{4 b}\right] F_{2: 1 ; 1}^{1: 2 ; 1}\left[\begin{array}{c}
\lambda+1: \lambda, 1 ; 1 \\
b+1,2: \lambda+1 ; 1
\end{array} \mid-\frac{x^{2}}{4},-\frac{x^{2}}{4}\right] \\
& +\frac{x_{0}^{2 b} \Gamma(\lambda+1) \exp \left(\frac{x_{0}^{2}}{4}\right)}{2 b(-1)^{b} 2^{2 b-1} \lambda \Gamma(b){ }_{1} F_{1}\left(\lambda, b,-\frac{x^{2}}{4}\right){ }_{1} F_{1}\left(\lambda, b,-\frac{x_{0}^{2}}{4}\right)} \\
& \times\left[{ }_{1} F_{1}\left(\lambda, b,-\frac{x^{2}}{4}\right) U\left(\lambda, b,-\frac{x_{0}^{2}}{4}\right)-{ }_{1} F_{1}\left(\lambda, b,-\frac{x_{0}^{2}}{4}\right) U\left(\lambda, b,-\frac{x^{2}}{4}\right)\right] \\
& \times\left\{{ }_{1} F_{1}\left(\lambda, b,-\frac{x_{0}^{2}}{4}\right){ }_{1} F_{1}\left(\lambda+1, b+1,-\frac{x_{0}^{2}}{4}\right)-\lambda{ }_{1} F_{1}\left(\lambda+1, b+1,-\frac{x_{0}^{2}}{4}\right)\right. \\
& \times\left[-\frac{x_{0}^{2}}{4 b}\right] F_{2: 1 ; 1}^{1: 2 ; 1}\left[\begin{array}{c}
\lambda+1: \lambda, 1 ; 1 \\
b+1,2: \lambda+1 ; 1
\end{array} \mid-\frac{x_{0}^{2}}{4},-\frac{x_{0}^{2}}{4}\right]+\lambda_{1} F_{1}\left(\lambda, b,-\frac{x_{0}^{2}}{4}\right) \\
& \left.\times\left[-\frac{x_{0}^{2}}{4 b}\right] \quad F_{2: 1 ; 1}^{1: 2 ; 1}\left[\begin{array}{cc}
\lambda+2: \lambda+1,1 ; 1 \\
b+2,2: \lambda+2 ; 1
\end{array} \mid-\frac{x_{0}^{2}}{4},-\frac{x_{0}^{2}}{4}\right]\right\} \text {. }
\end{aligned}
$$


To the best of our knowledge the integral formula (28) has not been stated yet in the literature. We omit to show further examples that involve more general forms of the integrand because evaluation of (10) would result in very long expressions.

Example: Bessel functions. Let us choose $\lambda=1 / 2, b=1$ and $x_{0}=0$. Upon substituting these values in our formula (28), we obtain the integral formula

$$
\int_{0}^{x} \int_{0}^{x_{1}} \frac{x_{2} I_{0}\left(\frac{x_{2}^{2}}{8}\right)^{2}}{x_{1} I_{0}\left(\frac{x_{1}^{2}}{8}\right)^{2}} d x_{2} d x_{1}=\frac{x^{2} \exp \left(\frac{x^{2}}{8}\right)}{4 I_{0}\left(\frac{x^{2}}{8}\right)} F_{2: 1 ; 1}^{1: 2 ; 1}\left[\begin{array}{c}
\frac{3}{2}: \frac{1}{2}, 1 ; 1 \\
2,2: \frac{3}{2} ; 1
\end{array} \mid-\frac{x^{2}}{4},-\frac{x^{2}}{4}\right],
$$

where $I_{0}$ stands for the modified Bessel function of the first kind. As in the case of the general result (28), we were unable to find identity (29) in integral tables. Now, in order to verify correctness of this result through numerical integration, let us now evaluate (29) for the specific value $x=1$. Upon substitution into the right side of (29) we get

$$
\frac{\exp \left(\frac{1}{8}\right)}{4 I_{0}\left(\frac{1}{8}\right)} \quad F_{2: 1 ; 1}^{1: 2 ; 1}\left[\begin{array}{cr}
\frac{3}{2}: \frac{1}{2}, 1 ; 1 \\
2,2: & \frac{3}{2} \quad ; 1
\end{array} \mid-\frac{1}{4},-\frac{1}{4}\right]=0.249567
$$

This coincides with the value that we obtain if we integrate the left side of (29) numerically.

Example: error function. We apply the parameter setting $\lambda=1 / 2, b=3 / 2$ and $x_{0}=0$ in our formula (28). After simplifying we obtain the integral identity

$\int_{0}^{x} \int_{0}^{x_{1}} \exp \left(-\frac{x_{1}^{2}}{4}+\frac{x_{2}^{2}}{4}\right) \frac{\operatorname{erf}\left(\frac{x_{2}}{2}\right)^{2}}{\operatorname{erf}\left(\frac{x_{1}}{2}\right)^{2}} d x_{2} d x_{1}=\frac{x^{3}}{6 \sqrt{\pi} \operatorname{erf}\left(\frac{x}{2}\right)} F_{2: 1 ; 1}^{1: 2 ; 1}\left[\begin{array}{c}\frac{3}{2}: \frac{1}{2}, 1 ; 1 \\ \frac{5}{2}, 2: \frac{3}{2} ; 1\end{array} \mid-\frac{x^{2}}{4},-\frac{x^{2}}{4}\right]$.

Evaluation of this expression for $x=1$ gives

$$
\frac{1}{6 \sqrt{\pi} \operatorname{erf}\left(\frac{1}{2}\right)} F_{2: 1 ; 1}^{1: 2 ; 1}\left[\begin{array}{cc|}
\frac{3}{2}: \frac{1}{2}, 1 ; 1 & \frac{5}{2}, 2 ; 1
\end{array}-\frac{1}{4},-\frac{1}{4}\right]=0.163764
$$

A direct numerical integration confirms this result.

\section{$5 \quad$ Hypergeometric integrals}

The purpose of this section is the construction of integrals that involve hypergeometric functions. Therefore, our starting point is the hypergeometric equation [1]

$$
z(1-z) \psi^{\prime \prime}(z)+[c-(a+b+1) z] \psi^{\prime}(z)-a b \psi(z)=0
$$

where the constants $a, b$ and $c$ are real-valued. Equation (30) has the following general solution

$$
\psi(z)=c_{12} F_{1}(a, b, c, z)+c_{2} z^{1-c}{ }_{2} F_{1}(a-c+1, b-c+1,2-c, z) .
$$

Here, ${ }_{2} F_{1}$ denotes the hypergeometric function [1], imposing the restriction that $c$ is not a nonpositive integer. Furthermore, the constants $c_{1}$ and $c_{2}$ are arbitrary. Our integral formulas (7) and (10) become applicable once equation (30) is brought to the form (6). This can be achieved by means of the function

$$
y(x)=\sqrt{\frac{1}{z^{\prime}(x)}} z(x)^{\frac{c}{2}}[z(x)-1]^{\frac{1}{2}(a+b-c+1)} \psi[z(x)],
$$


introducing a smooth coordinate change $z$ and a solution $\psi$ of the hypergeometric equation (30). Similar to the case of (14), our function (32) is a solution to the equation

$$
y^{\prime \prime}(x)+W(x) y(x)=0,
$$

where $W$ stands for the following expression

$$
\begin{aligned}
W(x) & =\frac{z^{\prime}(x)^{2}\left\{c(2-c)+z(x)\left[-4 a b+2 c(a+b-1)+z(x)-(a-b)^{2} z(x)\right]\right\}}{4 z(x)[z(x)-1]^{2}} \\
& -\frac{1}{z^{\prime}(x)^{2}}\left[\frac{3}{4} z^{\prime \prime}(x)^{2}-\frac{z^{\prime \prime \prime}(x) z^{\prime}(x)}{2[z(x)-1]}\right] .
\end{aligned}
$$

The general solution of the transformed equation (33) is given by (32) after substitution of (31). We obtain the following result

$$
\begin{aligned}
y(x) & =\sqrt{\frac{1}{z^{\prime}(x)}} z(x)^{\frac{c}{2}}[z(x)-1]^{\frac{1}{2}(a+b-c+1)}\left\{c_{1}{ }_{2} F_{1}[a, b, c, z(x)]\right. \\
& \left.+c_{2} z(x)^{1-c}{ }_{2} F_{1}[a-c+1, b-c+1,2-c, z(x)]\right\}
\end{aligned}
$$

The remaining task consists in matching the general equation (33) with the required form (14). To this end, our function $W$ in (34) has to be rewritten as $W=\lambda-V$ for a constant $\lambda$ and a function $V$. The latter form of $W$ is only attained if the coordinate change $z$ is chosen in an appropriate way, as the following examples show.

\subsection{Single integration}

In order to apply our formula (7) for single integration, we will now introduce parameter settings that convert our equation (33) into a special case of (14). To this end, let us now implement the following definitions.

$$
z(x)=\cosh ^{2}(x) \quad a=-\frac{\sqrt{-\lambda}}{2} \quad b=\frac{\sqrt{-\lambda}}{2} .
$$

Let us point out that $\lambda$ must be negative or zero in order for $a$ and $b$ to attain real values, as requested for (30). We will comment on this below when processing examples. Upon substitution into (34), our function $W$ reads

$$
W(x)=\lambda+\left(\frac{1}{4}-c^{2}\right) \frac{1}{\sinh ^{2}(x)}+\left[\frac{3}{4}+c(c-2)\right] \frac{1}{\cosh ^{2}(x)} .
$$

This expression matches the form $W=\lambda-V$ if the function $V$ is chosen as

$$
V(x)=-\left(\frac{1}{4}-c^{2}\right) \frac{1}{\sinh ^{2}(x)}-\left[\frac{3}{4}+c(c-2)\right] \frac{1}{\cosh ^{2}(x)} .
$$

Since we have shown that (36) renders (33) as a particular case of (14), the function (35) can now be used in our integral formula (7). The latter function has the following explicit form

$$
\begin{aligned}
y(x) & =\sinh ^{\frac{1}{2}-c}(x) \cosh ^{-\frac{1}{2}+c}(x)\left\{c_{1}{ }_{2} F_{1}\left[-\frac{\sqrt{-\lambda}}{2}, \frac{\sqrt{-\lambda}}{2}, c, \cosh ^{2}(x)\right]\right. \\
& \left.+c_{2} \cosh ^{2-2 c}(x)_{2} F_{1}\left[-\frac{\sqrt{-\lambda}}{2}-c+1, \frac{\sqrt{-\lambda}}{2}-c+1,2-c, \cosh ^{2}(x)\right]\right\} .
\end{aligned}
$$


In order to keep subsequent calculations manageable, we introduce further settings $c_{1}=1, c_{2}=$ 0. After renaming $y(x)=u(x, \lambda)$, we obtain from (37)

$$
u(x, \lambda)=\sinh ^{\frac{1}{2}-c}(x) \cosh ^{-\frac{1}{2}+c}(x)_{2} F_{1}\left[-\frac{\sqrt{-\lambda}}{2}, \frac{\sqrt{-\lambda}}{2}, c, \cosh ^{2}(x)\right] .
$$

We will apply our integral formula (7) to this function. In order to obtain a closed-form representation of the integral, we evaluate the right side of the latter formula. For the sake of brevity we will resort to the abbreviations in (36) for the parameters $a$ and $b$. We obtain the Wronskian in the form

$$
\begin{aligned}
& W_{u, \frac{\partial u}{\partial \lambda}}(x, \lambda)= \\
& \quad=\frac{1}{4 c} \sinh ^{2-2 c}(x) \cosh ^{2 c}(x){ }_{2} F_{1}\left[a+1, b+1, c+1, \cosh ^{2}(x)\right]\left\{2{ }_{2} F_{1}\left[a, b, c, \cosh ^{2}(x)\right]\right. \\
& \quad-2 \lambda \frac{\partial}{\partial \lambda}{ }_{2} F_{1}\left[a, b, c, \cosh ^{2}(x)\right]+2 \lambda \frac{{ }_{2} F_{1}\left[a, b, c, \cosh ^{2}(x)\right]}{{ }_{2} F_{1}\left[a+1, b+1, c+1, \cosh ^{2}(x)\right]} \\
& \left.\quad \times \frac{\partial}{\partial \lambda}{ }_{2} F_{1}\left[a+1, b+1, c+1, \cosh ^{2}(x)\right]\right\} .
\end{aligned}
$$

We observe that the expression on the right side contains partial derivatives of the hypergeometric function that can be written in terms of $\mathrm{KdF}$ functions. To this end, we apply the chain rule and afterwards implement identities (4) and (5). This gives

$$
\begin{aligned}
& \frac{\partial}{\partial \lambda}{ }_{2} F_{1}\left[a, b, c, \cosh ^{2}(x)\right] \\
& =\frac{1}{4 \sqrt{-\lambda}} \frac{\partial}{\partial a}{ }_{2} F_{1}\left[a, b, c, \cosh ^{2}(x)\right]-\frac{1}{4 \sqrt{-\lambda}} \frac{\partial}{\partial b}{ }_{2} F_{1}\left[a, b, c, \cosh ^{2}(x)\right] \\
& =\frac{1}{8 c} \cosh ^{2}(x) \quad F_{2: 1 ; 1}^{2: 1 ; 2}\left[\begin{array}{ccc}
a+1, b+1: & 1 & ; a, 1 \\
c+1,2 & : a+1 ; & 1
\end{array} \mid \cosh ^{2}(x), \cosh ^{2}(x)\right] \\
& -\frac{\sqrt{-\lambda}}{16 c^{2}} \cosh ^{2}(x) \quad F_{2: 1 ; 1}^{2: 1 ; 2}\left[\begin{array}{ccc|c}
a+1, b+1: & 1 & ; b, 1 \\
c+1,2 & : b+1 ; & \cosh ^{2}(x), \cosh ^{2}(x)
\end{array}\right] \text {. }
\end{aligned}
$$

We observe that the two derivatives in (39) are applied to slightly different hypergeometric functions. Since the difference between these functions lies only in shifted indices, we can adjust expression (40) in a straightforward manner. Upon substituting the expanded derivatives into 
the Wronskian (39), we arrive at its final form

$$
\begin{aligned}
& W_{u, \frac{\partial u}{\partial \lambda}}(x, \lambda) \\
& =\frac{1}{4 c}\left\{\sinh ^{2-2 c}(x) \cosh ^{2 c}(x){ }_{2} F_{1}\left[a+1, b+1, c+1, \cosh ^{2}(x)\right]{ }_{2} F_{1}\left[a, b, c, \cosh ^{2}(x)\right]\right\} \\
& +\frac{1}{4 c}\left\{\sinh ^{2-2 c}(x) \cosh ^{2 c}(x){ }_{2} F_{1}\left[a+1, b+1, c+1, \cosh ^{2}(x)\right]_{2} F_{1}\left[a, b, c, \cosh ^{2}(x)\right]\right\} \\
& -\frac{\lambda}{2 c}\left\{\sinh ^{2-2 c}(x) \cosh ^{2 c}(x){ }_{2} F_{1}\left[a+1, b+1, c+1, \cosh ^{2}(x)\right] \times\right. \\
& \times\left\{\frac{1}{8 c} \cosh ^{2}(x) \quad F_{2: 1 ; 1}^{2: 1 ; 2}\left[\begin{array}{ccc}
a+1, b+1: & 1 & ; a, 1 \\
c+1,2 & : a+1 ; 1
\end{array} \mid \cosh ^{2}(x), \cosh ^{2}(x)\right]\right. \\
& \left.\left.-\frac{\sqrt{-\lambda}}{16 c^{2}} \cosh ^{2}(x) \quad F_{2: 1 ; 1}^{2: 1 ; 2}\left[\begin{array}{ccc|}
a+1, b+1: & 1 & ; b, 1 \\
c+1,2 & : b+1 ; & 1
\end{array} \mid \cosh ^{2}(x), \cosh ^{2}(x)\right]\right\}\right\} \\
& +\frac{\lambda}{2 c}\left\{\sinh ^{2-2 c}(x) \cosh ^{2 c}(x){ }_{2} F_{1}\left[a, b, c, \cosh ^{2}(x)\right]\right. \\
& \times\left\{\frac{1}{8 c} \cosh ^{2}(x) \quad F_{2: 1 ; 1}^{2: 1 ; 2}\left[\begin{array}{ccc|c}
a+2, b+2: & 1 & ; a+1,1 \\
c+2,2 & : a+2 ; & 1
\end{array} \mid \cosh ^{2}(x), \cosh ^{2}(x)\right]\right. \\
& \left.-\frac{\sqrt{-\lambda}}{16 c^{2}} \cosh ^{2}(x) \quad F_{2: 1 ; 1}^{2: 1 ; 2}\left[\begin{array}{ccc|c}
a+2, b+2: & 1 & ; b+1,1 & \cosh ^{2}(x), \cosh ^{2}(x) \\
c+2,2 & : b+2 ; & 1 &
\end{array}\right\}\right\} .
\end{aligned}
$$

Now that we have computed the Wronskian, we can set up our integral formula (7) for the present case. Upon substitution of (38) into its left side, we obtain

$$
\int_{x_{0}}^{x} \sinh ^{1-2 c}(t) \cosh ^{-1+2 c}(t){ }_{2} F_{1}\left[-\frac{\sqrt{-\lambda}}{2}, \frac{\sqrt{-\lambda}}{2}, c, \cosh ^{2}(t)\right]^{2} d t=\left.W_{u, \frac{\partial u}{\partial \lambda}}(t, \lambda)\right|_{x} ^{x_{0}},
$$

where the Wronskians must be replaced by the expression obtained in (41).

Example: elliptic integral. Let us now consider the parameter setting $\lambda=-1$ and $c=1$. Recall that a negative value of $\lambda$ guarantees the constants $a$ and $b$ in (36). Substitution into the left side of (28) gives

$$
\int_{x_{0}}^{x} \sinh ^{-1}(t) \cosh (t){ }_{2} F_{1}\left[-\frac{\sqrt{-1}}{2}, \frac{\sqrt{-1}}{2}, 1, \cosh ^{2}(t)\right]^{2} d t=\int_{x_{0}}^{x} \frac{4 \cosh (t)}{\pi^{2} \sinh (t)} E\left[\cosh ^{2}(t)\right]^{2} d t .
$$

We see that the hypergeometric function degenerates to a complete elliptic integral [1], denoted by $E$. Upon evaluation of our formula (42) for the integration limits $x_{0}=1, x=2$ we obtain by substitution into (41)

$$
\int_{1}^{2} \frac{4 \cosh (t)}{\pi^{2} \sinh (t)} E\left[\cosh ^{2}(t)\right]^{2} d t=\left.W_{u, \frac{\partial u}{\partial \lambda}}(t,-1)\right|_{2} ^{1}=-1.68269+0.532137 i .
$$

It is straightforward to verify that this result can be recovered by direct numerical integration. 
Example: hyperbolic tangent. We will now see that our formula (42) can yield a representation for an integral of elementary hyperbolic functions. To this end, let us consider the following identity $[9]$

$$
\int_{x_{0}}^{x} \tanh ^{p}(t) d t=-\left.\frac{\tanh ^{p-1}(t)}{p-1}\right|_{x_{0}} ^{x}+\int_{x_{0}}^{x} \tanh ^{p-2}(t) d t, \quad p \neq 1 .
$$

If $p$ is a positive integer, then iteration of this identity allows to resolve the integral in terms of a finite sum. Let us now focus on the case where $p$ is an arbitrary real number, but not integer. We employ the parameter settings $\lambda=0$ and $c=(1-p) / 2$ on the left side of (42). This gives

$$
\int_{x_{0}}^{x} \sinh ^{p}(t) \cosh ^{-p}(t){ }_{2} F_{1}\left[0,0, \frac{1-p}{2}, \cosh ^{2}(t)\right]^{2} d t=\int_{x_{0}}^{x} \tanh ^{p}(t) d t .
$$

As in the previous example, let us mention that our setting $\lambda=0$ implies $a=b=0$ in (36). We observe that the integral on the left side of (44) is a particular case of its counterpart in (42). As such, our formula (42) becomes applicable. Upon substitution of the current parameter settings into (41) and (42), the latter formula simplifies as follows

$$
\int_{x_{0}}^{x} \tanh ^{p}(t) d t=\left.\frac{\sinh ^{p+1}(t) \cosh ^{1-p}(t)}{1-p}{ }_{2} F_{1}\left[1,1, \frac{3-p}{2}, \cosh ^{2}(t)\right]\right|_{x} ^{x_{0}} .
$$

Hence, we obtain a closed-form representation for the integral on the left side of (45) that is valid for any value of the exponent $p$. This includes the case $p=1$ if the evaluation in (45) is understood in the sense of a limit. Note that due to our choice of parameters, no KdF function from (41) is present anymore. Let us now verify our identity (45) for the exponent $p=\sqrt{2}$ and the integration limits $x_{0}=1, x=2$.

$$
\int_{1}^{2} \tanh ^{\sqrt{2}}(t) d t=\left.\frac{\sinh ^{\sqrt{2}+1}(t) \cosh ^{1-\sqrt{2}}(t)}{1-\sqrt{2}}{ }_{2} F_{1}\left[1,1, \frac{3-\sqrt{2}}{2}, \cosh ^{2}(t)\right]\right|_{2} ^{1}=0.850747 .
$$

This value coincides with the result of numerical integration.

\subsection{Double integration}

We will now present an example of double integration involving hypergeometric functions. To this end, we use identity (10) and the function $u$ defined in (38). Before we can start our construction, we need a solution $v$ of (33), such that $u$ and $v$ are linearly independent. This function $v$ can be obtained from the general form (35) by applying the settings $c_{1}=0$ and $c_{2}=1$

$$
v(x, \lambda)=\sinh ^{\frac{1}{2}-c}(x) \cosh ^{\frac{3}{2}-c}(x){ }_{2} F_{1}\left[1-c-\frac{\sqrt{-\lambda}}{2}, 1-c+\frac{\sqrt{-\lambda}}{2}, 2-c, \cosh ^{2}(x)\right] .
$$

We are now ready to evaluate the right side of (10) by substituting (38) and (46). Since the resulting expressions will become very large, we will perform the evaluation termwise. Since the first two terms are identical up to the value of $x$, it is sufficient to compute one of them. We find

$$
\begin{aligned}
& \frac{1}{u(x, \lambda)} \frac{\partial}{\partial \lambda} u(x, \lambda)= \\
& =\frac{1}{4 \sqrt{-\lambda}{ }_{2} F_{1}\left[a, b, c, \cosh ^{2}(x)\right]}\left\{\frac{\partial}{\partial a}{ }_{2} F_{1}\left[a, b, c, \cosh ^{2}(x)\right]-\frac{\partial}{\partial b}{ }_{2} F_{1}\left[a, b, c, \cosh ^{2}(x)\right]\right\},
\end{aligned}
$$


where the abbreviations $a$ and $b$ from (36) are used to shorten the notation. We can now replace the partial derivatives of the hypergeometric function by means of our relations (4) and (5). After some simplification we arrive at

$$
\begin{aligned}
& \frac{1}{u(x, \lambda)} \frac{\partial}{\partial \lambda} u(x, \lambda) \\
& =\frac{1}{4 \sqrt{-\lambda}{ }_{2} F_{1}\left[a, b, c, \cosh ^{2}(x)\right]} \\
& \times\left\{\frac{\sqrt{-\lambda}}{2 c} \cosh ^{2}(x) \quad F_{2: 1 ; 1}^{2: 1 ; 2}\left[\begin{array}{ccc}
a+1, b+1: & 1 \quad ; a, 1 \\
c+1,2 & : a+1 ; & 1
\end{array} \mid \cosh ^{2}(x), \cosh ^{2}(x)\right]\right. \\
& \left.+\frac{\lambda}{4 c^{2}} \cosh ^{2}(x) \quad F_{2: 1 ; 1}^{2: 1 ; 2}\left[\begin{array}{ccc|c}
a+1, b+1: & 1 & ; b, 1 & \cosh ^{2}(x), \cosh ^{2}(x) \\
c+1,2 & : b+1 ; & 1
\end{array}\right]\right\} \text {. }
\end{aligned}
$$

Next, we evaluate the Wronskian of $u$ and its partial derivative with respect to $\lambda$. This gives

$$
\begin{aligned}
W_{u, \frac{\partial u}{\partial \lambda}}(x, \lambda) & =-\frac{1}{\sqrt{-\lambda}} \sinh ^{2-2 c}(x) \cosh ^{-2+2 c}(x){ }_{2} F_{1}\left[a, b, c, \cosh ^{2}(x)\right] \\
& \times\left\{{ }_{2} F_{1}\left[a, b, c, \cosh ^{2}(x)\right]-{ }_{2} F_{1}\left[a+1, b, c, \cosh ^{2}(x)\right]\right\} \\
& +\frac{\lambda}{2 c} \sinh ^{2-2 c}(x) \cosh ^{2 c}(x){ }_{2} F_{1}\left[a, b, c, \cosh ^{2}(x)\right] \\
& \times\left\{\frac{1}{4 \sqrt{-\lambda}} \frac{\partial}{\partial a}{ }_{2} F_{1}\left[a+1, b+1, c+1, \cosh ^{2}(x)\right]\right. \\
& \left.-\frac{1}{4 \sqrt{-\lambda}} \frac{\partial}{\partial b}{ }_{2} F_{1}\left[a+1, b+1, c+1, \cosh ^{2}(x)\right]\right\} \\
& -\sqrt{-\lambda} \sinh ^{2-2 c}(x) \cosh ^{-2+2 c}(x){ }_{2} F_{1}\left[a, b, c, \cosh ^{2}(x)\right] \\
& \times\left\{\frac{1}{4 \sqrt{-\lambda}} \frac{\partial}{\partial a}{ }_{2} F_{1}\left[a, b, c, \cosh ^{2}(x)\right]-\frac{1}{4 \sqrt{-\lambda}} \frac{\partial}{\partial b}{ }_{2} F_{1}\left[a, b, c, \cosh ^{2}(x)\right]\right\} \\
& -\sqrt{-\lambda} \sinh ^{2-2 c}(x) \cosh ^{-2+2 c}(x){ }_{2} F_{1}\left[a+1, b, c, \cosh ^{2}(x)\right] \\
& \times\left\{\frac{1}{4 \sqrt{-\lambda}} \frac{\partial}{\partial a}{ }_{2} F_{1}\left[a, b, c, \cosh ^{2}(x)\right]-\frac{1}{4 \sqrt{-\lambda}} \frac{\partial}{\partial b}{ }_{2} F_{1}\left[a, b, c, \cosh ^{2}(x)\right]\right\} .
\end{aligned}
$$

We can express the partial derivatives of the hypergeometric functions by means of KdF functions. Upon using our identities (4) and (5), we obtain

$$
\begin{aligned}
W_{u, \frac{\partial u}{\partial \lambda}}(x, \lambda) & =-\frac{1}{\sqrt{-\lambda}} \sinh ^{2-2 c}(x) \cosh ^{-2+2 c}(x)_{2} F_{1}\left[a, b, c, \cosh ^{2}(x)\right] \\
& \times\left\{{ }_{2} F_{1}\left[a, b, c, \cosh ^{2}(x)\right]-{ }_{2} F_{1}\left[a+1, b, c, \cosh ^{2}(x)\right]\right\} \\
& +\frac{\lambda}{2 c} \sinh ^{2-2 c}(x) \cosh ^{2 c}(x){ }_{2} F_{1}\left[a, b, c, \cosh ^{2}(x)\right]
\end{aligned}
$$




$$
\begin{aligned}
& \times\left\{\frac{1}{8 c} \cosh ^{2}(x) F_{2: 1 ; 1}^{2: 1 ; 2}\left[\begin{array}{ccc|c}
a+2, b+2: & 1 & ; a+1,1 \\
c+2,2 & : a+2 & ; & 1
\end{array} \mid \cosh ^{2}(x), \cosh ^{2}(x)\right]\right. \\
& -\frac{\sqrt{-\lambda}}{16 c^{2}} \cosh ^{2}(x) F_{2: 1 ; 1}^{2: 1 ; 2}\left[\begin{array}{ccc|c}
a+2, b+2: & 1 & ; b+1,1 & \cosh ^{2}(x), \cosh ^{2}(x) \\
c+2,2 & : b+2 & 1 & 1
\end{array}\right\} \\
& -\sqrt{-\lambda} \sinh ^{2-2 c}(x) \cosh ^{-2+2 c}(x){ }_{2} F_{1}\left[a, b, c, \cosh ^{2}(x)\right] \\
& \times\left\{\frac{1}{8 c} \cosh ^{2}(x) F_{2: 1 ; 1}^{2: 1 ; 2}\left[\begin{array}{ccc}
a+1, b+1: & 1 ; a, 1 \\
c+1,2 & : a+1 ; 1
\end{array} \mid \cosh ^{2}(x), \cosh ^{2}(x)\right]\right. \\
& \left.-\frac{\sqrt{-\lambda}}{16 c^{2}} \cosh ^{2}(x) \quad F_{2: 1 ; 1}^{2: 1 ; 2}\left[\begin{array}{ccc|c}
a+1, b+1: & 1 & ; b, 1 & \cosh ^{2}(x), \cosh ^{2}(x) \\
c+1,2 & : b+1 ; & 1
\end{array}\right]\right\} \\
& -\sqrt{-\lambda} \sinh ^{2-2 c}(x) \cosh ^{-2+2 c}(x){ }_{2} F_{1}\left[a+1, b, c, \cosh ^{2}(x)\right] \\
& \times\left\{\frac{1}{8 c} \cosh ^{2}(x) \quad F_{2: 1 ; 1}^{2: 1 ; 2}\left[\begin{array}{ccc|c}
a+1, b+1: & 1 & ; a, 1 \\
c+1,2 & : a+1 ; & 1
\end{array} \mid \cosh ^{2}(x), \cosh ^{2}(x)\right]\right. \\
& \left.-\frac{\sqrt{-\lambda}}{16 c^{2}} \cosh ^{2}(x) F_{2: 1 ; 1}^{2: 1 ; 2}\left[\begin{array}{ccc|c}
a+1, b+1: & 1 & ; b, 1 & \cosh ^{2}(x), \cosh ^{2}(x) \\
c+1,2 & : b+1 ; & 1
\end{array}\right]\right\} \text {. }
\end{aligned}
$$

It remains to find an expression for the Wronskian of the functions (38) and (46). A straightforward calculation yields

$$
\begin{aligned}
& W_{u, v}(\lambda)=2 \sinh ^{2-2 c}(x) \\
& \quad \times\left\{(1-c+a){ }_{2} F_{1}\left[a, b, c, \cosh ^{2}(x)\right]_{2} F_{1}\left[2-c+a, 1-c+b, 2-c, \cosh ^{2}(x)\right]\right. \\
& \left.-a_{2} F_{1}\left[a+1, b, c, \cosh ^{2}(x)\right]_{2} F_{1}\left[1-c+a, 1-c+b, 2-c, \cosh ^{2}(x)\right]\right\} .
\end{aligned}
$$

Note that the values of this Wronskian do not depend on $x$, even though $x$ appears in the above representation. If we inspect the right side of our integral formula (10), we observe that in the present case all quantities have now been determined. After substitution of (38), the latter formula reads

$$
\begin{aligned}
& \int_{x_{0}}^{x} \int_{x_{0}}^{x_{1}} \frac{\cosh ^{-1+2 c}\left(x_{2}\right) \sinh ^{1-2 c}\left(x_{2}\right){ }_{2} F_{1}\left[-\frac{\sqrt{-\lambda}}{2}, \frac{\sqrt{-\lambda}}{2}, c, \cosh ^{2}\left(x_{2}\right)\right]^{2}}{\cosh ^{-1+2 c}\left(x_{1}\right) \sinh ^{1-2 c}\left(x_{1}\right){ }_{2} F_{1}\left[-\frac{\sqrt{-\lambda}}{2}, \frac{\sqrt{-\lambda}}{2}, c, \cosh ^{2}\left(x_{1}\right)\right]^{2}} d x_{2} d x_{1} \\
& =\frac{1}{u\left(x_{0}, \lambda\right)} \frac{\partial}{\partial \lambda} u\left(x_{0}, \lambda\right)-\frac{1}{u(x, \lambda)} \frac{\partial}{\partial \lambda} u(x, \lambda)+\frac{W_{u, \frac{\partial u}{\partial \lambda}}\left(x_{0}, \lambda\right)}{W_{u, v}(\lambda)}\left[\frac{v(x, \lambda)}{u(x, \lambda)}-\frac{v\left(x_{0}, \lambda\right)}{u\left(x_{0}, \lambda\right)}\right],
\end{aligned}
$$

where the right side has to be calculated by inserting (38), (46), (47), (48) and (49). Due to the length of the involved expressions we omit to show the full form of (50), but state a few special cases.

Example: elliptic integral. We will now generalize our example (43) to the case of double integration. To this end, let us substitute the parameter values $\lambda=-1$ and $c=1$ into the left 
side of (50). This gives

$$
\begin{aligned}
\int_{x_{0}}^{x} \int_{x_{0}}^{x_{1}} \frac{\cosh \left(x_{2}\right) \sinh ^{-1}\left(x_{2}\right){ }_{2} F_{1}\left[-\frac{\sqrt{1}}{2}, \frac{\sqrt{1}}{2}, 1, \cosh ^{2}\left(x_{2}\right)\right]^{2}}{\cosh \left(x_{1}\right) \sinh ^{-1}\left(x_{1}\right){ }_{2} F_{1}\left[-\frac{\sqrt{1}}{2}, \frac{\sqrt{1}}{2}, 1, \cosh ^{2}\left(x_{1}\right)\right]^{2}} d x_{2} d x_{1} \\
=\int_{x_{0}} \int_{x_{0}}^{x_{1}} \frac{\tanh \left(x_{1}\right) E\left[\cosh ^{2}\left(x_{2}\right)\right]^{2}}{\tanh \left(x_{2}\right) E\left[\cosh ^{2}\left(x_{1}\right)\right]^{2}} d x_{2} d x_{1},
\end{aligned}
$$

recall that $E$ stands for the complete elliptic integral. After plugging our parameter values into (50), we obtain a closed-form representation for the integral on the right side of (51). Since the resulting expression is very long, we omit to show it here. Instead, we use (51) to evaluate our formula (50) for the integration limits $x_{0}=1$ and $x=2$. We obtain

$$
\begin{aligned}
\int_{1}^{2} \int_{1}^{x_{1}} \frac{\tanh \left(x_{1}\right) E\left[\cosh ^{2}\left(x_{2}\right)\right]^{2}}{\tanh \left(x_{2}\right) E\left[\cosh ^{2}\left(x_{1}\right)\right]^{2}} d x_{2} d x_{1} \\
=\frac{1}{u(1,-1)}\left[\frac{\partial}{\partial \lambda} u(1, \lambda)\right]_{\mid \lambda=-1}-\frac{1}{u(2,-1)}\left[\frac{\partial}{\partial \lambda} u(2, \lambda)\right]_{\mid \lambda=-1} \\
+\frac{W_{u, \frac{\partial u}{\partial \lambda}}(1,-1)}{W_{u, v}(\lambda)}\left[\frac{v(2,-1)}{u(2,-1)}-\frac{v(1,-1)}{u(1,-1)}\right] \\
=0.248226-0.0574144 i
\end{aligned}
$$

As in the previous examples it is straightforward to check this result through numerical integration.

Example: Gegenbauer polynomials. Hypergeometric functions can degenerate to different kinds of polynomials, a particular case of which are the Gegenbauer polynomials [1]. In order to obtain such a case, we set $c=1 / 2$ and reparametrize $\lambda=-4 n^{2}$ for a nonnegative integer $n$. Substitution into the left side of (50) gives

$$
\int_{x_{0}}^{x} \int_{x_{0}}^{x_{1}} \frac{{ }_{2} F_{1}\left[-n, n, \frac{1}{2}, \cosh ^{2}\left(x_{2}\right)\right]^{2}}{F_{1}\left[-n, n, \frac{1}{2}, \cosh ^{2}\left(x_{1}\right)\right]^{2}} d x_{2} d x_{1}=\int_{x_{0}}^{x} \int_{x_{0}}^{x_{1}} \frac{C_{n}\left[-\cosh ^{2}\left(2 x_{2}\right)\right]^{2}}{C_{n}\left[-\cosh ^{2}\left(2 x_{1}\right)\right]^{2}} d x_{2} d x_{1},
$$

where $C$ stands for a Gegenbauer polynomial. We can now use our formula (50) to provide a representation of the integral on the right side of (52). Insertion of explicit values $x_{0}=1, x=2$ and $n=3$ yields

$$
\begin{aligned}
& \int_{1}^{2} \int_{1}^{x_{1}} \frac{C_{3}\left[-\cosh ^{2}\left(2 x_{2}\right)\right]^{2}}{C_{3}\left[-\cosh ^{2}\left(2 x_{1}\right)\right]^{2}} d x_{2} d x_{1} \\
& =\frac{1}{u(1,-9)}\left[\frac{\partial}{\partial \lambda} u(1, \lambda)\right]_{\mid \lambda=-9}-\frac{1}{u(2,-9)}\left[\frac{\partial}{\partial \lambda} u(2, \lambda)\right]_{\mid \lambda=-9} \\
& +\frac{W_{u, \frac{\partial u}{\partial \lambda}}(1,-9)}{W_{u, v}(\lambda)}\left[\frac{v(2,-9)}{u(2,-9)}-\frac{v(1,-9)}{u(1,-9)}\right] \\
& =-8.48897
\end{aligned}
$$


Observe that $n=3$ corresponds to $\lambda=-9$. Correctness of the result (53) is verified by numerical integration.

\section{Concluding remarks}

We have shown that our identities (7), (10) can be used to generate closed-form representations of confluent hypergeometric and hypergeometric integrals in terms of KdF functions. The examples presented in this work are far from being exhaustive. First of all, we observe that equations (11) and (30) can be taken into the required form (6) not only by means of the coordinate changes provided in (17) and (36), respectively. Instead, there are infinitely many possibilities of such coordinate changes, each of which yields new integrands in (7) and (10). Furthermore, the latter formulas are applicable to equations that are not of hypergeometric type, such as Mathieu-type or spheroidal equations. As a final generalization of our work let us mention the existence of counterparts to (10) that involve multiple integrals of even order. Exploration of these topics is subject to future research.

\section{References}

[1] M. Abramowitz and I.A. Stegun, "Handbook of Mathematical Functions with Formulas, Graphs, and Mathematical Tables", Dover, 1964

[2] L.U. Ancarani and G. Gasaneo, "Derivatives of any order of the hypergeometric function ${ }_{p} F_{q}\left(a_{1}, \ldots, a_{p} ; b_{1}, \ldots, b_{q} ; z\right)$ with respect to the parameters $a_{i}$ and $b_{i} "$, J. Phys. A 43 (2010), 085210

[3] L.U. Ancarani and G. Gasaneo, "Derivatives of any order of the Gaussian hypergeometric function ${ }_{2} F_{1}(a, b, c ; z)$ with respect to the parameters $a, b$ and $c$ ", J. Phys. A 42 (2009), 395208

[4] L.U. Ancarani and G. Gasaneo, "Derivatives of any order of the confluent hypergeometric function ${ }_{1} F_{1}(a, b, z)$ with respect to the parameter $a$ or $b$ ", J. Math. Phys. 49 (2008), 063508

[5] W.W. Bell, "Special functions for scientists and engineers", (Dover Publications, Mineola, USA, 2004)

[6] A. Contreras-Astorga and A. Schulze-Halberg, "On integral and differential representations of Jordan chains and the confluent supersymmetry algorithm", J. Phys. A 48 (2015), 315202

[7] D. Cvijovic and A.R. Miller, "A reduction formula for the Kampé de Fériet function", Appl. Math. Lett. 23 (2010), 769-771

[8] H. Exton, "Multiple Hypergeometric Functions and Applications", (Ellis Horwood Limited, Chichester, UK, 1976)

[9] I.S. Gradshteyn and I.M. Ryzhik, "Table of Integrals, Series and Products", (Academic Press, London, UK, 2014)

[10] E. Kamke, "Differentialgleichungen - Lösungsmethoden und Lösungen", (B.G. Teubner, Stuttgart, 1983)

[11] A.F. Nikiforov and V.B. Uvarov, "Special Functions of Mathematical Physics: a Unified Introduction with Applications", (Springer Science and Business Media, Basel, Switzerland, 2013) 
[12] A. Schulze-Halberg, "Arbitrary-order Jordan chains associated with quantum-mechanical Hamiltonians: representations and integral formulas", J. Math. Phys. 57 (2016), 023521

[13] J.B. Seaborn, "Hypergeometric Functions and Their Applications", (Springer Science and Business Media, New York, USA, 2011)

[14] H.M. Srivastava and P.W. Karlsson, "Multiple Gaussian Hypergeometric Series", (Ellis Horwood Limited, Chichester, UK, 1985)

[15] D. Zwillinger, "CRC Standard Mathematical Tables and Formulae", Advances in Applied Mathematics, 32 ed., (CRC Press, Boca Raton, USA, 2012)

[16] http://www.wolframalpha.com, http://functions.wolfram.com 